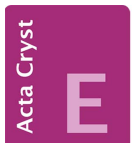
COMMUNICATIONS

ISSN 2056-9890

\section{Crystal structure of 2-butylsulfanyl-4,6- bis[(E)-styryl]pyrimidine}

\section{Aijian Wang* and Guanghui Li}

China-Australia Joint Research Center for Functional Molecular Materials, Scientific Research Academy \& School of Chemistry and Chemical Engineering, Jiangsu University, Zhenjiang 212013, People's Republic of China. *Correspondence e-mail: wajujs@ujs.edu.cn

Received 22 April 2015; accepted 24 April 2015

Edited by W. T. A. Harrison, University of Aberdeen, Scotland

In the title compound, $\mathrm{C}_{24} \mathrm{H}_{24} \mathrm{~N}_{2} \mathrm{~S}$, the dihedral angles between the central pyrimidine ring and pendant benzene rings are $18.46(6)$ and $5.95(6)^{\circ}$. The butylsulfanyl side chain adopts a twisted conformation $\left[\mathrm{S}-\mathrm{C}-\mathrm{C}-\mathrm{C}=177.34(10)^{\circ}\right.$ and $\left.\mathrm{C}-\mathrm{C}-\mathrm{C}-\mathrm{C}=67.68(18)^{\circ}\right]$. No directional interactions beyond typical van der Waals contacts could be identified in the crystal.

Keywords: crystal structure; weak interactions; pyrimidine.

CCDC reference: 1010472

\section{Related literature}

For general background to pyrimidine derivatives and their applications, see: Walker et al. (2009); van Laar et al. (2001); Casas et al. (2006); Deng et al. (2008); Nguyen (2008). For the synthesis of the title compound, see: Liu et al. (2007).

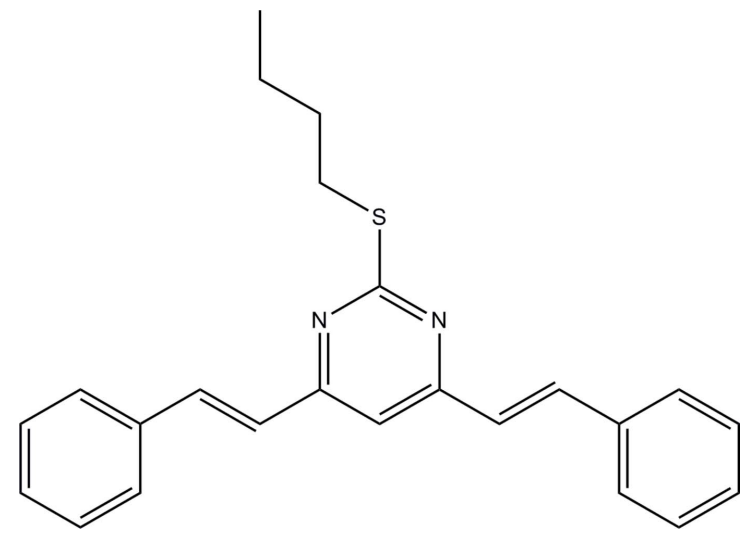

\section{Experimental}

2.1. Crystal data

$\mathrm{C}_{24} \mathrm{H}_{24} \mathrm{~N}_{2} \mathrm{~S}$

$M_{r}=372.51$

Monoclinic, $P 2_{1} / c$

$a=9.0447(18) \AA$

$b=9.3798(19) \AA$

$c=23.802(7) \AA$

$\beta=103.44(3)^{\circ}$

$V=1964.0(8) \AA^{3}$

$Z=4$

Mo $K \alpha$ radiation

$\mu=0.18 \mathrm{~mm}^{-1}$

$T=153 \mathrm{~K}$

$0.26 \times 0.23 \times 0.22 \mathrm{~mm}$

\subsection{Data collection}

Rigaku Saturn 724+ CCD diffractometer

Absorption correction: multi-scan multi-scan $T_{\min }=0.830, T_{\max }=1.000$

9602 measured reflections 3487 independent reflections 3193 reflections with $I>2 \sigma(I)$ $R_{\text {int }}=0.020$

\subsection{Refinement}

$R\left[F^{2}>2 \sigma\left(F^{2}\right)\right]=0.034$

$w R\left(F^{2}\right)=0.087$

$S=1.06$

3487 reflections

245 parameters

$\mathrm{H}$-atom parameters constrained

$\Delta \rho_{\max }=0.20{\mathrm{e} \AA^{-3}}^{-3}$

$\Delta \rho_{\min }=-0.23$ e $\AA^{-3}$

Data collection: CrystalClear (Rigaku, 2008); cell refinement: CrystalClear; data reduction: CrystalClear; program(s) used to solve structure: SHELXTL (Sheldrick, 2008); program(s) used to refine structure: SHELXTL; molecular graphics: SHELXTL; software used to prepare material for publication: SHELXTL.

\section{Acknowledgements}

This research was supported financially by the Research Foundation of Jiangsu University (grant No. 13JDG066).

Supporting information for this paper is available from the IUCr electronic archives (Reference: HB7413).

\section{References}

Casas, J. S., Castellano, E. E., Couce, M. D., Ellena, J., Sánchez, A., Sordo, J. \& Taboada, C. (2006). J. Inorg. Biochem. 91, 1858-1860.

Deng, Y., Wang, Y., Cherian, C., Hou, Z., Buck, S. A., Matherly, L. H. \& Gangjee, A. (2008). J. Med. Chem. 51, 5052-5063.

Laar, M. van, Volkerts, E. \& Verbaten, M. (2001). Psychopharmacology, 154 189-197.

Liu, B., Hu, X.-L., Liu, J., Zhao, Y.-D. \& Huang, Z.-L. (2007). Tetrahedron Lett. 48, 5958-5962.

Nguyen, T. L. (2008). Anticancer Agents Med. Chem. 8, 710-716.

Rigaku (2008). CrystalClear. Rigaku Corporation, Tokyo, Japan.

Sheldrick, G. M. (2008). Acta Cryst. A64, 112-122.

Walker, S. R., Carter, E. J., Huff, B. C. \& Morris, J. C. (2009). Chem. Rev. 109 3080-3098. 


\section{supporting information}

Acta Cryst. (2015). E71, o368 [https://doi.org/10.1107/S2056989015008166]

Crystal structure of 2-butylsulfanyl-4,6-bis[(E)-styryl] pyrimidine

Aijian Wang and Guanghui Li

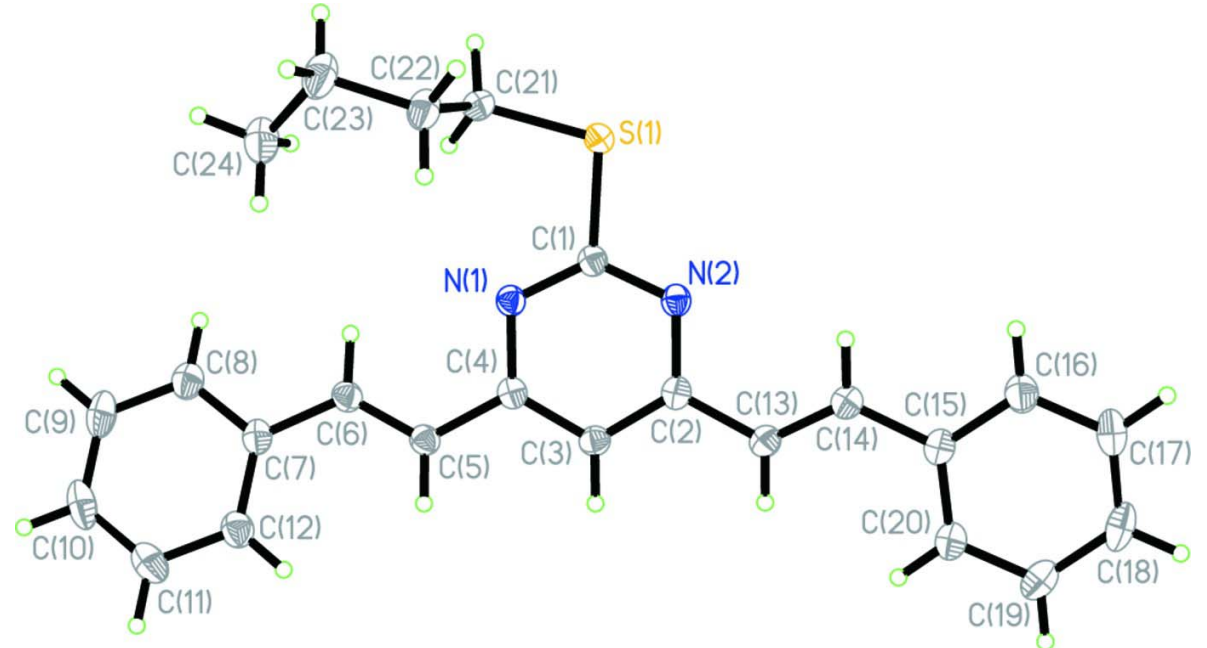

Figure 1

The molecular structure of (I) showing 50\% displacement ellipsoids.

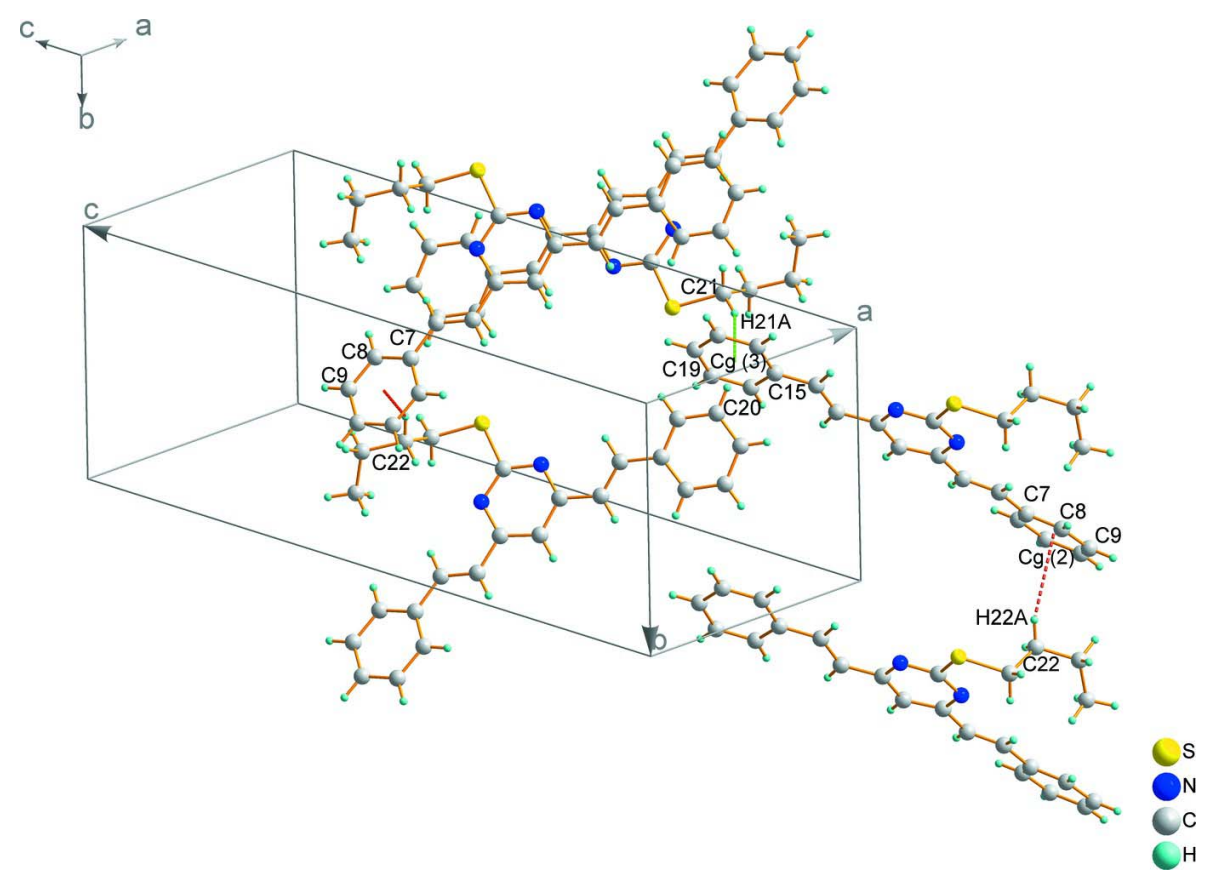


Figure 2

Packing diagram for (I).

2-Butylsulfanyl-4,6-bis[(E)-styryl]pyrimidine

Crystal data

$\mathrm{C}_{24} \mathrm{H}_{24} \mathrm{~N}_{2} \mathrm{~S}$

$M_{r}=372.51$

Monoclinic, $P 2{ }_{1} / c$

$a=9.0447$ (18) $\AA$

$b=9.3798(19) \AA$

$c=23.802(7) \AA$

$\beta=103.44(3)^{\circ}$

$V=1964.0(8) \AA^{3}$

$Z=4$

\section{Data collection}

Rigaku CCD

diffractometer

Radiation source: fine-focus sealed tube

Graphite monochromator

phi and $\omega$ scans

Absorption correction: multi-scan multi-scan

$T_{\min }=0.830, T_{\max }=1.000$

\section{Refinement}

Refinement on $F^{2}$

Least-squares matrix: full

$R\left[F^{2}>2 \sigma\left(F^{2}\right)\right]=0.034$

$w R\left(F^{2}\right)=0.087$

$S=1.06$

3487 reflections

245 parameters

0 restraints

Primary atom site location: structure-invariant direct methods
$F(000)=792$

$D_{\mathrm{x}}=1.260 \mathrm{Mg} \mathrm{m}^{-3}$

Mo $K \alpha$ radiation, $\lambda=0.71073 \AA$

Cell parameters from 4606 reflections

$\theta=3.9-28.6^{\circ}$

$\mu=0.18 \mathrm{~mm}^{-1}$

$T=153 \mathrm{~K}$

Prism, yellow

$0.26 \times 0.23 \times 0.22 \mathrm{~mm}$

9602 measured reflections

3487 independent reflections

3193 reflections with $I>2 \sigma(I)$

$R_{\text {int }}=0.020$

$\theta_{\max }=25.2^{\circ}, \theta_{\min }=3.9^{\circ}$

$h=-10 \rightarrow 8$

$k=-10 \rightarrow 11$

$l=-28 \rightarrow 28$

Secondary atom site location: difference Fourier map

Hydrogen site location: inferred from neighbouring sites

$\mathrm{H}$-atom parameters constrained

$w=1 /\left[\sigma^{2}\left(F_{\mathrm{o}}^{2}\right)+(0.0468 P)^{2}+0.4259 P\right]$

where $P=\left(F_{\mathrm{o}}{ }^{2}+2 F_{\mathrm{c}}{ }^{2}\right) / 3$

$(\Delta / \sigma)_{\max }=0.002$

$\Delta \rho_{\max }=0.20 \mathrm{e} \AA^{-3}$

$\Delta \rho_{\min }=-0.23$ e $\AA^{-3}$

Special details

Geometry. All e.s.d.'s (except the e.s.d. in the dihedral angle between two 1.s. planes) are estimated using the full covariance matrix. The cell e.s.d.'s are taken into account individually in the estimation of e.s.d.'s in distances, angles and torsion angles; correlations between e.s.d.'s in cell parameters are only used when they are defined by crystal symmetry. An approximate (isotropic) treatment of cell e.s.d.'s is used for estimating e.s.d.'s involving 1.s. planes.

Refinement. Refinement of $F^{2}$ against ALL reflections. The weighted $R$-factor $w R$ and goodness of fit $S$ are based on $F^{2}$, conventional $R$-factors $R$ are based on $F$, with $F$ set to zero for negative $F^{2}$. The threshold expression of $F^{2}>\sigma\left(F^{2}\right)$ is used only for calculating $R$-factors (gt) etc. and is not relevant to the choice of reflections for refinement. $R$-factors based on $F^{2}$ are statistically about twice as large as those based on $F$, and $R$ - factors based on ALL data will be even larger.

Fractional atomic coordinates and isotropic or equivalent isotropic displacement parameters $\left(\hat{A}^{2}\right)$

\begin{tabular}{lllll}
\hline & $x$ & $y$ & $z$ & $U_{\text {iso }} * / U_{\text {eq }}$ \\
\hline S1 & $0.14918(4)$ & $0.26493(4)$ & $0.884447(14)$ & $0.02603(12)$ \\
N1 & $0.29497(11)$ & $0.49599(11)$ & $0.93583(4)$ & $0.0198(2)$
\end{tabular}




\begin{tabular}{|c|c|c|c|c|}
\hline $\mathrm{N} 2$ & $0.14298(11)$ & $0.35823(11)$ & 0.98633 (4) & $0.0202(2)$ \\
\hline $\mathrm{C} 1$ & $0.20397(13)$ & $0.38866(13)$ & $0.94166(5)$ & $0.0193(3)$ \\
\hline $\mathrm{C} 2$ & $0.17542(13)$ & $0.45128(13)$ & $1.03058(5)$ & $0.0194(3)$ \\
\hline $\mathrm{C} 3$ & $0.26563(13)$ & $0.57051(13)$ & $1.02829(5)$ & $0.0205(3)$ \\
\hline H3 & 0.2852 & 0.6377 & 1.0590 & $0.025^{*}$ \\
\hline $\mathrm{C} 4$ & $0.32650(13)$ & $0.58934(13)$ & $0.98031(5)$ & $0.0191(3)$ \\
\hline $\mathrm{C} 5$ & $0.42814(13)$ & $0.70891(13)$ & $0.97653(5)$ & $0.0204(3)$ \\
\hline H5 & 0.4634 & 0.7661 & 1.0099 & $0.025^{*}$ \\
\hline C6 & 0.47367 (14) & $0.74153(13)$ & $0.92880(6)$ & $0.0219(3)$ \\
\hline H6 & 0.4330 & 0.6851 & 0.8957 & $0.026^{*}$ \\
\hline $\mathrm{C} 7$ & $0.57954(14)$ & $0.85485(13)$ & $0.92161(6)$ & $0.0225(3)$ \\
\hline $\mathrm{C} 8$ & $0.60175(15)$ & $0.88109(15)$ & $0.86649(6)$ & $0.0281(3)$ \\
\hline H8 & 0.5451 & 0.8285 & 0.8346 & $0.034^{*}$ \\
\hline C9 & $0.70538(16)$ & $0.98295(16)$ & $0.85754(7)$ & $0.0343(4)$ \\
\hline H9 & 0.7194 & 0.9993 & 0.8197 & $0.041^{*}$ \\
\hline $\mathrm{C} 10$ & $0.78780(16)$ & $1.06029(15)$ & $0.90348(7)$ & $0.0353(4)$ \\
\hline H10 & 0.8589 & 1.1298 & 0.8974 & $0.042 *$ \\
\hline $\mathrm{C} 11$ & $0.76658(16)$ & $1.03641(15)$ & $0.95844(7)$ & $0.0332(3)$ \\
\hline H11 & 0.8230 & 1.0901 & 0.9901 & $0.040^{*}$ \\
\hline $\mathrm{C} 12$ & $0.66348(15)$ & $0.93468(14)$ & $0.96766(6)$ & $0.0273(3)$ \\
\hline H12 & 0.6498 & 0.9192 & 1.0056 & $0.033^{*}$ \\
\hline $\mathrm{C} 13$ & $0.11375(13)$ & $0.42439(14)$ & $1.08114(5)$ & $0.0210(3)$ \\
\hline H13 & 0.1239 & 0.4973 & 1.1095 & $0.025^{*}$ \\
\hline $\mathrm{C} 14$ & $0.04425(14)$ & $0.30423(14)$ & $1.08999(5)$ & $0.0222(3)$ \\
\hline H14 & 0.0362 & 0.2324 & 1.0613 & $0.027 *$ \\
\hline $\mathrm{C} 15$ & $-0.02107(14)$ & $0.27106(14)$ & $1.13927(5)$ & $0.0218(3)$ \\
\hline C16 & $-0.09987(15)$ & $0.14320(15)$ & $1.13976(6)$ & $0.0286(3)$ \\
\hline H16 & -0.1076 & 0.0781 & 1.1086 & $0.034^{*}$ \\
\hline $\mathrm{C} 17$ & $-0.16687(16)$ & $0.10953(16)$ & $1.18474(7)$ & $0.0341(3)$ \\
\hline H17 & -0.2208 & 0.0224 & 1.1841 & $0.041^{*}$ \\
\hline $\mathrm{C} 18$ & $-0.15537(16)$ & $0.20253(17)$ & $1.23059(6)$ & $0.0327(3)$ \\
\hline H18 & -0.2010 & 0.1794 & 1.2616 & $0.039^{*}$ \\
\hline C19 & $-0.07708(15)$ & $0.32952(16)$ & $1.23120(6)$ & $0.0302(3)$ \\
\hline H19 & -0.0692 & 0.3936 & 1.2627 & $0.036^{*}$ \\
\hline $\mathrm{C} 20$ & $-0.01020(15)$ & $0.36359(15)$ & $1.18626(6)$ & $0.0265(3)$ \\
\hline $\mathrm{H} 20$ & 0.0438 & 0.4508 & 1.1873 & $0.032^{*}$ \\
\hline $\mathrm{C} 21$ & $0.25447(15)$ & $0.32567(15)$ & $0.83313(5)$ & $0.0252(3)$ \\
\hline $\mathrm{H} 21 \mathrm{~A}$ & 0.2058 & 0.2874 & 0.7945 & $0.030^{*}$ \\
\hline $\mathrm{H} 21 \mathrm{~B}$ & 0.2489 & 0.4310 & 0.8308 & $0.030^{*}$ \\
\hline $\mathrm{C} 22$ & $0.42011(15)$ & $0.28109(15)$ & $0.84837(6)$ & $0.0286(3)$ \\
\hline $\mathrm{H} 22 \mathrm{~A}$ & 0.4260 & 0.1761 & 0.8524 & $0.034^{*}$ \\
\hline $\mathrm{H} 22 \mathrm{~B}$ & 0.4702 & 0.3232 & 0.8861 & $0.034^{*}$ \\
\hline $\mathrm{C} 23$ & $0.50566(18)$ & $0.32712(16)$ & $0.80329(7)$ & $0.0370(4)$ \\
\hline $\mathrm{H} 23 \mathrm{~A}$ & 0.6071 & 0.2814 & 0.8124 & $0.044^{*}$ \\
\hline H23B & 0.4501 & 0.2916 & 0.7650 & $0.044^{*}$ \\
\hline $\mathrm{C} 24$ & $0.52647(17)$ & $0.48708(16)$ & $0.79936(7)$ & $0.0364(4)$ \\
\hline $\mathrm{H} 24 \mathrm{~A}$ & 0.5838 & 0.5078 & 0.7701 & $0.055^{*}$ \\
\hline H24B & 0.5823 & 0.5235 & 0.8369 & $0.055^{*}$ \\
\hline
\end{tabular}




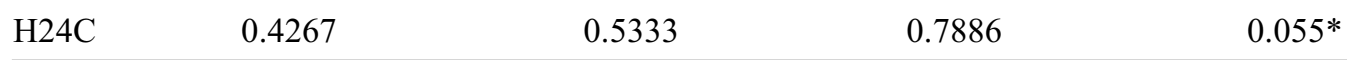

Atomic displacement parameters $\left(\AA^{2}\right)$

\begin{tabular}{lllllll}
\hline & $U^{11}$ & $U^{22}$ & $U^{33}$ & $U^{12}$ & $U^{13}$ & $U^{23}$ \\
\hline S1 & $0.0269(2)$ & $0.0267(2)$ & $0.0270(2)$ & $-0.00895(13)$ & $0.01143(15)$ & $-0.00718(14)$ \\
N1 & $0.0190(5)$ & $0.0189(6)$ & $0.0221(5)$ & $0.0003(4)$ & $0.0062(4)$ & $0.0002(4)$ \\
N2 & $0.0189(5)$ & $0.0199(6)$ & $0.0226(5)$ & $0.0010(4)$ & $0.0066(4)$ & $0.0013(4)$ \\
C1 & $0.0174(6)$ & $0.0188(6)$ & $0.0219(6)$ & $0.0015(5)$ & $0.0051(5)$ & $0.0001(5)$ \\
C2 & $0.0164(6)$ & $0.0205(7)$ & $0.0214(6)$ & $0.0038(5)$ & $0.0045(5)$ & $0.0033(5)$ \\
C3 & $0.0208(6)$ & $0.0202(7)$ & $0.0206(6)$ & $0.0011(5)$ & $0.0048(5)$ & $-0.0007(5)$ \\
C4 & $0.0169(6)$ & $0.0185(6)$ & $0.0216(6)$ & $0.0028(5)$ & $0.0042(5)$ & $0.0015(5)$ \\
C5 & $0.0203(6)$ & $0.0180(6)$ & $0.0231(6)$ & $-0.0003(5)$ & $0.0052(5)$ & $-0.0008(5)$ \\
C6 & $0.0230(7)$ & $0.0187(6)$ & $0.0248(7)$ & $-0.0007(5)$ & $0.0071(5)$ & $-0.0010(5)$ \\
C7 & $0.0218(6)$ & $0.0184(6)$ & $0.0292(7)$ & $0.0026(5)$ & $0.0096(5)$ & $0.0023(5)$ \\
C8 & $0.0299(7)$ & $0.0268(7)$ & $0.0299(7)$ & $0.0007(6)$ & $0.0116(6)$ & $0.0030(6)$ \\
C9 & $0.0355(8)$ & $0.0322(8)$ & $0.0410(9)$ & $0.0035(6)$ & $0.0207(7)$ & $0.0129(7)$ \\
C10 & $0.0260(7)$ & $0.0243(8)$ & $0.0595(10)$ & $-0.0016(6)$ & $0.0178(7)$ & $0.0089(7)$ \\
C11 & $0.0267(7)$ & $0.0249(7)$ & $0.0471(9)$ & $-0.0038(6)$ & $0.0069(7)$ & $-0.0020(7)$ \\
C12 & $0.0270(7)$ & $0.0235(7)$ & $0.0327(7)$ & $-0.0010(5)$ & $0.0094(6)$ & $0.0005(6)$ \\
C13 & $0.0195(6)$ & $0.0226(7)$ & $0.0210(6)$ & $0.0024(5)$ & $0.0051(5)$ & $0.0007(5)$ \\
C14 & $0.0226(6)$ & $0.0226(7)$ & $0.0217(6)$ & $0.0023(5)$ & $0.0058(5)$ & $0.0013(5)$ \\
C15 & $0.0186(6)$ & $0.0241(7)$ & $0.0231(7)$ & $0.0038(5)$ & $0.0056(5)$ & $0.0048(5)$ \\
C16 & $0.0299(7)$ & $0.0266(7)$ & $0.0306(7)$ & $-0.0012(6)$ & $0.0099(6)$ & $0.0026(6)$ \\
C17 & $0.0311(8)$ & $0.0331(8)$ & $0.0411(8)$ & $-0.0036(6)$ & $0.0148(7)$ & $0.0108(7)$ \\
C18 & $0.0291(8)$ & $0.0413(9)$ & $0.0324(8)$ & $0.0077(6)$ & $0.0171(6)$ & $0.0142(7)$ \\
C19 & $0.0322(7)$ & $0.0361(8)$ & $0.0240(7)$ & $0.0080(6)$ & $0.0102(6)$ & $0.0040(6)$ \\
C20 & $0.0277(7)$ & $0.0271(7)$ & $0.0261(7)$ & $0.0008(6)$ & $0.0092(6)$ & $0.0043(6)$ \\
C21 & $0.0269(7)$ & $0.0296(7)$ & $0.0195(6)$ & $-0.0036(6)$ & $0.0066(5)$ & $-0.0028(6)$ \\
C22 & $0.0297(7)$ & $0.0272(7)$ & $0.0324(8)$ & $0.0029(6)$ & $0.0146(6)$ & $0.0036(6)$ \\
C23 & $0.0427(9)$ & $0.0314(8)$ & $0.0454(9)$ & $0.0054(7)$ & $0.0276(7)$ & $0.0035(7)$ \\
C24 & $0.0350(8)$ & $0.0343(8)$ & $0.0436(9)$ & $-0.0003(6)$ & $0.0171(7)$ & $0.0063(7)$
\end{tabular}

Geometric parameters $(\AA, \stackrel{o}{)}$

\begin{tabular}{llll}
\hline $\mathrm{S} 1-\mathrm{C} 1$ & $1.7701(13)$ & $\mathrm{C} 13-\mathrm{H} 13$ & 0.9500 \\
$\mathrm{~S} 1-\mathrm{C} 21$ & $1.8066(14)$ & $\mathrm{C} 14-\mathrm{C} 15$ & $1.4642(18)$ \\
$\mathrm{N} 1-\mathrm{C} 1$ & $1.3279(16)$ & $\mathrm{C} 14-\mathrm{H} 14$ & 0.9500 \\
$\mathrm{~N} 1-\mathrm{C} 4$ & $1.3523(16)$ & $\mathrm{C} 15-\mathrm{C} 16$ & $1.3966(19)$ \\
$\mathrm{N} 2-\mathrm{C} 1$ & $1.3375(16)$ & $\mathrm{C} 15-\mathrm{C} 20$ & $1.4009(19)$ \\
$\mathrm{N} 2-\mathrm{C} 2$ & $1.3466(16)$ & $\mathrm{C} 16-\mathrm{C} 17$ & $1.384(2)$ \\
$\mathrm{C} 2-\mathrm{C} 3$ & $1.3929(18)$ & $\mathrm{C} 16-\mathrm{H} 16$ & 0.9500 \\
$\mathrm{C} 2-\mathrm{C} 13$ & $1.4611(18)$ & $\mathrm{C} 17-\mathrm{C} 18$ & $1.382(2)$ \\
$\mathrm{C} 3-\mathrm{C} 4$ & $1.3896(17)$ & $\mathrm{C} 17-\mathrm{H} 17$ & 0.9500 \\
$\mathrm{C} 3-\mathrm{H} 3$ & 0.9500 & $\mathrm{C} 18-\mathrm{C} 19$ & $0.384(2)$ \\
$\mathrm{C} 4-\mathrm{C} 5$ & $1.4662(17)$ & $\mathrm{C} 18-\mathrm{H} 18$ & $1.3831(19)$ \\
$\mathrm{C} 5-\mathrm{C} 6$ & $1.3309(18)$ & $\mathrm{C} 19-\mathrm{C} 20$ & 0.9500 \\
$\mathrm{C} 5-\mathrm{H} 5$ & 0.9500 & $\mathrm{C} 19-\mathrm{H} 19$ &
\end{tabular}




\begin{tabular}{|c|c|c|c|}
\hline $\mathrm{C} 6-\mathrm{C} 7$ & $1.4670(18)$ & $\mathrm{C} 20-\mathrm{H} 20$ & 0.9500 \\
\hline $\mathrm{C} 6-\mathrm{H} 6$ & 0.9500 & $\mathrm{C} 21-\mathrm{C} 22$ & $1.5160(19)$ \\
\hline $\mathrm{C} 7-\mathrm{C} 8$ & $1.3946(19)$ & $\mathrm{C} 21-\mathrm{H} 21 \mathrm{~A}$ & 0.9900 \\
\hline $\mathrm{C} 7-\mathrm{C} 12$ & $1.3969(19)$ & $\mathrm{C} 21-\mathrm{H} 21 \mathrm{~B}$ & 0.9900 \\
\hline $\mathrm{C} 8-\mathrm{C} 9$ & $1.389(2)$ & $\mathrm{C} 22-\mathrm{C} 23$ & $1.5239(19)$ \\
\hline $\mathrm{C} 8-\mathrm{H} 8$ & 0.9500 & $\mathrm{C} 22-\mathrm{H} 22 \mathrm{~A}$ & 0.9900 \\
\hline $\mathrm{C} 9-\mathrm{C} 10$ & $1.378(2)$ & $\mathrm{C} 22-\mathrm{H} 22 \mathrm{~B}$ & 0.9900 \\
\hline $\mathrm{C} 9-\mathrm{H} 9$ & 0.9500 & $\mathrm{C} 23-\mathrm{C} 24$ & $1.518(2)$ \\
\hline $\mathrm{C} 10-\mathrm{C} 11$ & $1.384(2)$ & $\mathrm{C} 23-\mathrm{H} 23 \mathrm{~A}$ & 0.9900 \\
\hline $\mathrm{C} 10-\mathrm{H} 10$ & 0.9500 & $\mathrm{C} 23-\mathrm{H} 23 \mathrm{~B}$ & 0.9900 \\
\hline $\mathrm{C} 11-\mathrm{C} 12$ & $1.3868(19)$ & $\mathrm{C} 24-\mathrm{H} 24 \mathrm{~A}$ & 0.9800 \\
\hline $\mathrm{C} 11-\mathrm{H} 11$ & 0.9500 & $\mathrm{C} 24-\mathrm{H} 24 \mathrm{~B}$ & 0.9800 \\
\hline $\mathrm{C} 12-\mathrm{H} 12$ & 0.9500 & $\mathrm{C} 24-\mathrm{H} 24 \mathrm{C}$ & 0.9800 \\
\hline $\mathrm{C} 13-\mathrm{C} 14$ & $1.3310(19)$ & & \\
\hline $\mathrm{C} 1-\mathrm{S} 1-\mathrm{C} 21$ & $102.48(6)$ & $\mathrm{C} 15-\mathrm{C} 14-\mathrm{H} 14$ & 116.6 \\
\hline $\mathrm{C} 1-\mathrm{N} 1-\mathrm{C} 4$ & $115.60(11)$ & $\mathrm{C} 16-\mathrm{C} 15-\mathrm{C} 20$ & $117.82(12)$ \\
\hline $\mathrm{C} 1-\mathrm{N} 2-\mathrm{C} 2$ & $115.35(11)$ & $\mathrm{C} 16-\mathrm{C} 15-\mathrm{C} 14$ & $119.39(12)$ \\
\hline $\mathrm{N} 1-\mathrm{C} 1-\mathrm{N} 2$ & $128.69(12)$ & $\mathrm{C} 20-\mathrm{C} 15-\mathrm{C} 14$ & $122.78(12)$ \\
\hline $\mathrm{N} 1-\mathrm{C} 1-\mathrm{S} 1$ & $119.09(9)$ & $\mathrm{C} 17-\mathrm{C} 16-\mathrm{C} 15$ & $121.23(13)$ \\
\hline $\mathrm{N} 2-\mathrm{C} 1-\mathrm{S} 1$ & $112.22(9)$ & $\mathrm{C} 17-\mathrm{C} 16-\mathrm{H} 16$ & 119.4 \\
\hline $\mathrm{N} 2-\mathrm{C} 2-\mathrm{C} 3$ & $120.87(11)$ & $\mathrm{C} 15-\mathrm{C} 16-\mathrm{H} 16$ & 119.4 \\
\hline $\mathrm{N} 2-\mathrm{C} 2-\mathrm{C} 13$ & $118.47(11)$ & $\mathrm{C} 18-\mathrm{C} 17-\mathrm{C} 16$ & $120.05(14)$ \\
\hline $\mathrm{C} 3-\mathrm{C} 2-\mathrm{C} 13$ & $120.66(12)$ & $\mathrm{C} 18-\mathrm{C} 17-\mathrm{H} 17$ & 120.0 \\
\hline $\mathrm{C} 4-\mathrm{C} 3-\mathrm{C} 2$ & $118.80(12)$ & $\mathrm{C} 16-\mathrm{C} 17-\mathrm{H} 17$ & 120.0 \\
\hline $\mathrm{C} 4-\mathrm{C} 3-\mathrm{H} 3$ & 120.6 & $\mathrm{C} 17-\mathrm{C} 18-\mathrm{C} 19$ & $119.73(13)$ \\
\hline $\mathrm{C} 2-\mathrm{C} 3-\mathrm{H} 3$ & 120.6 & $\mathrm{C} 17-\mathrm{C} 18-\mathrm{H} 18$ & 120.1 \\
\hline $\mathrm{N} 1-\mathrm{C} 4-\mathrm{C} 3$ & $120.63(11)$ & $\mathrm{C} 19-\mathrm{C} 18-\mathrm{H} 18$ & 120.1 \\
\hline $\mathrm{N} 1-\mathrm{C} 4-\mathrm{C} 5$ & $117.93(11)$ & $\mathrm{C} 20-\mathrm{C} 19-\mathrm{C} 18$ & $120.36(14)$ \\
\hline $\mathrm{C} 3-\mathrm{C} 4-\mathrm{C} 5$ & $121.43(12)$ & $\mathrm{C} 20-\mathrm{C} 19-\mathrm{H} 19$ & 119.8 \\
\hline $\mathrm{C} 6-\mathrm{C} 5-\mathrm{C} 4$ & $123.44(12)$ & $\mathrm{C} 18-\mathrm{C} 19-\mathrm{H} 19$ & 119.8 \\
\hline $\mathrm{C} 6-\mathrm{C} 5-\mathrm{H} 5$ & 118.3 & $\mathrm{C} 19-\mathrm{C} 20-\mathrm{C} 15$ & $120.80(13)$ \\
\hline $\mathrm{C} 4-\mathrm{C} 5-\mathrm{H} 5$ & 118.3 & $\mathrm{C} 19-\mathrm{C} 20-\mathrm{H} 20$ & 119.6 \\
\hline $\mathrm{C} 5-\mathrm{C} 6-\mathrm{C} 7$ & $127.30(12)$ & $\mathrm{C} 15-\mathrm{C} 20-\mathrm{H} 20$ & 119.6 \\
\hline $\mathrm{C} 5-\mathrm{C} 6-\mathrm{H} 6$ & 116.4 & $\mathrm{C} 22-\mathrm{C} 21-\mathrm{S} 1$ & $113.46(9)$ \\
\hline $\mathrm{C} 7-\mathrm{C} 6-\mathrm{H} 6$ & 116.4 & $\mathrm{C} 22-\mathrm{C} 21-\mathrm{H} 21 \mathrm{~A}$ & 108.9 \\
\hline $\mathrm{C} 8-\mathrm{C} 7-\mathrm{C} 12$ & $118.20(12)$ & $\mathrm{S} 1-\mathrm{C} 21-\mathrm{H} 21 \mathrm{~A}$ & 108.9 \\
\hline $\mathrm{C} 8-\mathrm{C} 7-\mathrm{C} 6$ & $118.57(12)$ & $\mathrm{C} 22-\mathrm{C} 21-\mathrm{H} 21 \mathrm{~B}$ & 108.9 \\
\hline $\mathrm{C} 12-\mathrm{C} 7-\mathrm{C} 6$ & $123.19(12)$ & $\mathrm{S} 1-\mathrm{C} 21-\mathrm{H} 21 \mathrm{~B}$ & 108.9 \\
\hline $\mathrm{C} 9-\mathrm{C} 8-\mathrm{C} 7$ & $121.04(14)$ & $\mathrm{H} 21 \mathrm{~A}-\mathrm{C} 21-\mathrm{H} 21 \mathrm{~B}$ & 107.7 \\
\hline $\mathrm{C} 9-\mathrm{C} 8-\mathrm{H} 8$ & 119.5 & $\mathrm{C} 21-\mathrm{C} 22-\mathrm{C} 23$ & $112.88(12)$ \\
\hline $\mathrm{C} 7-\mathrm{C} 8-\mathrm{H} 8$ & 119.5 & $\mathrm{C} 21-\mathrm{C} 22-\mathrm{H} 22 \mathrm{~A}$ & 109.0 \\
\hline $\mathrm{C} 10-\mathrm{C} 9-\mathrm{C} 8$ & $120.01(14)$ & $\mathrm{C} 23-\mathrm{C} 22-\mathrm{H} 22 \mathrm{~A}$ & 109.0 \\
\hline $\mathrm{C} 10-\mathrm{C} 9-\mathrm{H} 9$ & 120.0 & $\mathrm{C} 21-\mathrm{C} 22-\mathrm{H} 22 \mathrm{~B}$ & 109.0 \\
\hline $\mathrm{C} 8-\mathrm{C} 9-\mathrm{H} 9$ & 120.0 & $\mathrm{C} 23-\mathrm{C} 22-\mathrm{H} 22 \mathrm{~B}$ & 109.0 \\
\hline $\mathrm{C} 9-\mathrm{C} 10-\mathrm{C} 11$ & $119.78(13)$ & $\mathrm{H} 22 \mathrm{~A}-\mathrm{C} 22-\mathrm{H} 22 \mathrm{~B}$ & 107.8 \\
\hline $\mathrm{C} 9-\mathrm{C} 10-\mathrm{H} 10$ & 120.1 & $\mathrm{C} 24-\mathrm{C} 23-\mathrm{C} 22$ & $114.46(12)$ \\
\hline $\mathrm{C} 11-\mathrm{C} 10-\mathrm{H} 10$ & 120.1 & $\mathrm{C} 24-\mathrm{C} 23-\mathrm{H} 23 \mathrm{~A}$ & 108.6 \\
\hline
\end{tabular}




\begin{tabular}{|c|c|}
\hline $\mathrm{C} 10-\mathrm{C} 11-\mathrm{C} 12$ & $120.47(14)$ \\
\hline $\mathrm{C} 10-\mathrm{C} 11-\mathrm{H} 11$ & 119.8 \\
\hline $\mathrm{C} 12-\mathrm{C} 11-\mathrm{H} 11$ & 119.8 \\
\hline $\mathrm{C} 11-\mathrm{C} 12-\mathrm{C} 7$ & $120.50(13)$ \\
\hline $\mathrm{C} 11-\mathrm{C} 12-\mathrm{H} 12$ & 119.8 \\
\hline $\mathrm{C} 7-\mathrm{C} 12-\mathrm{H} 12$ & 119.8 \\
\hline $\mathrm{C} 14-\mathrm{C} 13-\mathrm{C} 2$ & $124.15(12)$ \\
\hline $\mathrm{C} 14-\mathrm{C} 13-\mathrm{H} 13$ & 117.9 \\
\hline $\mathrm{C} 2-\mathrm{C} 13-\mathrm{H} 13$ & 117.9 \\
\hline $\mathrm{C} 13-\mathrm{C} 14-\mathrm{C} 15$ & $126.89(13)$ \\
\hline $\mathrm{C} 13-\mathrm{C} 14-\mathrm{H} 14$ & 116.6 \\
\hline $\mathrm{C} 4-\mathrm{N} 1-\mathrm{C} 1-\mathrm{N} 2$ & $-2.18(18)$ \\
\hline $\mathrm{C} 4-\mathrm{N} 1-\mathrm{C} 1-\mathrm{S} 1$ & $177.30(9)$ \\
\hline $\mathrm{C} 2-\mathrm{N} 2-\mathrm{C} 1-\mathrm{N} 1$ & $1.89(18)$ \\
\hline $\mathrm{C} 2-\mathrm{N} 2-\mathrm{C} 1-\mathrm{S} 1$ & $-177.61(8)$ \\
\hline $\mathrm{C} 21-\mathrm{S} 1-\mathrm{C} 1-\mathrm{N} 1$ & $3.44(11)$ \\
\hline $\mathrm{C} 21-\mathrm{S} 1-\mathrm{C} 1-\mathrm{N} 2$ & $-177.00(9)$ \\
\hline $\mathrm{C} 1-\mathrm{N} 2-\mathrm{C} 2-\mathrm{C} 3$ & $0.51(16)$ \\
\hline $\mathrm{C} 1-\mathrm{N} 2-\mathrm{C} 2-\mathrm{C} 13$ & $-179.51(10)$ \\
\hline $\mathrm{N} 2-\mathrm{C} 2-\mathrm{C} 3-\mathrm{C} 4$ & $-2.32(17)$ \\
\hline $\mathrm{C} 13-\mathrm{C} 2-\mathrm{C} 3-\mathrm{C} 4$ & $177.70(11)$ \\
\hline $\mathrm{C} 1-\mathrm{N} 1-\mathrm{C} 4-\mathrm{C} 3$ & $0.06(16)$ \\
\hline $\mathrm{C} 1-\mathrm{N} 1-\mathrm{C} 4-\mathrm{C} 5$ & $179.18(10)$ \\
\hline $\mathrm{C} 2-\mathrm{C} 3-\mathrm{C} 4-\mathrm{N} 1$ & $2.01(17)$ \\
\hline $\mathrm{C} 2-\mathrm{C} 3-\mathrm{C} 4-\mathrm{C} 5$ & $-177.07(11)$ \\
\hline $\mathrm{N} 1-\mathrm{C} 4-\mathrm{C} 5-\mathrm{C} 6$ & $9.66(18)$ \\
\hline $\mathrm{C} 3-\mathrm{C} 4-\mathrm{C} 5-\mathrm{C} 6$ & $-171.24(12)$ \\
\hline $\mathrm{C} 4-\mathrm{C} 5-\mathrm{C} 6-\mathrm{C} 7$ & $-177.30(11)$ \\
\hline $\mathrm{C} 5-\mathrm{C} 6-\mathrm{C} 7-\mathrm{C} 8$ & $-173.89(12)$ \\
\hline $\mathrm{C} 5-\mathrm{C} 6-\mathrm{C} 7-\mathrm{C} 12$ & $8.4(2)$ \\
\hline $\mathrm{C} 12-\mathrm{C} 7-\mathrm{C} 8-\mathrm{C} 9$ & $0.54(19)$ \\
\hline $\mathrm{C} 6-\mathrm{C} 7-\mathrm{C} 8-\mathrm{C} 9$ & $-177.27(12)$ \\
\hline $\mathrm{C} 7-\mathrm{C} 8-\mathrm{C} 9-\mathrm{C} 10$ & $-0.2(2)$ \\
\hline
\end{tabular}

$\begin{array}{ll}\mathrm{C} 22-\mathrm{C} 23-\mathrm{H} 23 \mathrm{~A} & 108.6 \\ \mathrm{C} 24-\mathrm{C} 23-\mathrm{H} 23 \mathrm{~B} & 108.6 \\ \mathrm{C} 22-\mathrm{C} 23-\mathrm{H} 23 \mathrm{~B} & 108.6 \\ \mathrm{H} 23 \mathrm{~A}-\mathrm{C} 23-\mathrm{H} 23 \mathrm{~B} & 107.6 \\ \mathrm{C} 23-\mathrm{C} 24-\mathrm{H} 24 \mathrm{~A} & 109.5 \\ \mathrm{C} 23-\mathrm{C} 24-\mathrm{H} 24 \mathrm{~B} & 109.5 \\ \mathrm{H} 24 \mathrm{~A}-\mathrm{C} 24-\mathrm{H} 24 \mathrm{~B} & 109.5 \\ \mathrm{C} 23-\mathrm{C} 24-\mathrm{H} 24 \mathrm{C} & 109.5 \\ \mathrm{H} 24 \mathrm{~A}-\mathrm{C} 24-\mathrm{H} 24 \mathrm{C} & 109.5 \\ \mathrm{H} 24 \mathrm{~B}-\mathrm{C} 24-\mathrm{H} 24 \mathrm{C} & 109.5\end{array}$

$\mathrm{C} 8-\mathrm{C} 9-\mathrm{C} 10-\mathrm{C} 11 \quad-0.2(2)$

$\mathrm{C} 9-\mathrm{C} 10-\mathrm{C} 11-\mathrm{C} 12 \quad 0.3(2)$

$\mathrm{C} 10-\mathrm{C} 11-\mathrm{C} 12-\mathrm{C} 7 \quad 0.0(2)$

$\mathrm{C} 8-\mathrm{C} 7-\mathrm{C} 12-\mathrm{C} 11 \quad-0.42(19)$

$\mathrm{C} 6-\mathrm{C} 7-\mathrm{C} 12-\mathrm{C} 11 \quad 177.28$ (12)

$\mathrm{N} 2-\mathrm{C} 2-\mathrm{C} 13-\mathrm{C} 14 \quad 9.40$ (18)

$\mathrm{C} 3-\mathrm{C} 2-\mathrm{C} 13-\mathrm{C} 14 \quad-170.62$ (12)

$\mathrm{C} 2-\mathrm{C} 13-\mathrm{C} 14-\mathrm{C} 15 \quad-179.42(11)$

$\mathrm{C} 13-\mathrm{C} 14-\mathrm{C} 15-\mathrm{C} 16 \quad 175.44$ (12)

$\mathrm{C} 13-\mathrm{C} 14-\mathrm{C} 15-\mathrm{C} 20 \quad-3.6(2)$

$\mathrm{C} 20-\mathrm{C} 15-\mathrm{C} 16-\mathrm{C} 17 \quad 0.83(19)$

$\mathrm{C} 14-\mathrm{C} 15-\mathrm{C} 16-\mathrm{C} 17-178.22(12)$

$\mathrm{C} 15-\mathrm{C} 16-\mathrm{C} 17-\mathrm{C} 18 \quad-0.6(2)$

$\mathrm{C} 16-\mathrm{C} 17-\mathrm{C} 18-\mathrm{C} 19 \quad 0.2(2)$

$\mathrm{C} 17-\mathrm{C} 18-\mathrm{C} 19-\mathrm{C} 20 \quad-0.1(2)$

$\mathrm{C} 18-\mathrm{C} 19-\mathrm{C} 20-\mathrm{C} 15 \quad 0.4(2)$

$\mathrm{C} 16-\mathrm{C} 15-\mathrm{C} 20-\mathrm{C} 19 \quad-0.72(18)$

$\mathrm{C} 14-\mathrm{C} 15-\mathrm{C} 20-\mathrm{C} 19 \quad 178.30(12)$

$\mathrm{C} 1-\mathrm{S} 1-\mathrm{C} 21-\mathrm{C} 22 \quad 78.82(11)$

$\mathrm{S} 1-\mathrm{C} 21-\mathrm{C} 22-\mathrm{C} 23 \quad 177.34(10)$

$\mathrm{C} 21-\mathrm{C} 22-\mathrm{C} 23-\mathrm{C} 24 \quad 67.68(18)$ 